\title{
METASTASIS OF NEUROENDOCRINE TUMOR MIMICKING PRIMARY BREAST CANCER: CASE REPORT
}

Yuki Tany Hirakawa Vieira', Andre Luiz de Freitas Perina', Daniela Ferreira Vieira Vendramini', Thatyanne Cunha Esposito Gallo', Felipe Eduardo Martins Andrade ${ }^{1}$

${ }^{1}$ Hospital Sírio Libanês - São Paulo (SP), Brazil.

Introduction: Neuroendocrine tumor has an annual incidence of 2-5 cases/100,000 inhabitants, most of them asymptomatic, and may clinically present as carcinoid syndrome: facial flushing, diarrhea, and bronchospasm. It has a great tendency to metastasize to regional lymph nodes and liver, being unusual in the breast. Clinical case: A black 47-year-old woman without comorbidities presented a nodule with progressive growth for 2 years in the right upper inner quadrant (RUIQ), biopsied and diagnosed as breast cancer, without a specific subtype or immunohistochemistry (IHC). Physical examination revealed a $3 \mathrm{~cm}$ nodule, hard and fixed axillary lymph node, and enlarged yet fibroelastic and mobile anterior cervical lymph node. Ultrasound (US) identified two irregular nodules in the RUIQ and the junction of the right outer quadrants (JROQ) measuring $2.5 \mathrm{~cm}$ and $0.7 \mathrm{~cm}$, respectively, and level I axillary lymph node with cortical thickening. The JROQ nodule and the axillary lymph node were biopsied. The cervical lymph node did not show loss of hilum or suspicious abnormalities on US and was not biopsied. Anatomopathological results of the nodule were compatible with invasive carcinoma without a specific subtype, with estrogen receptor weakly positive (10\%), Ki-67 7\%, and negative for other markers. The axillary lymph node was negative for metastasis. During staging, an abdominal computed tomography identified a $1.9 \mathrm{~cm}$ lesion of likely neuroendocrine origin in the ileocecal valve with metastasis to the liver, regional lymph nodes, and breast. Complementary IHC of the biopsy slide was later performed with chromogranin, synaptophysin, and CDX-2, and the diagnosis reached was breast metastasis of neuroendocrine tumor. An external review of biopsy slides of the RUIQ nodule was requested, and the patient was referred to the oncology department to continue treatment. Conclusion: Metastasis of gastrointestinal tumors to the breast corresponds to less than $0.5 \%$ of cancers, with 15 reports in the literature, of which only 7 were asymptomatic, and their clinical presentation started with breast lesion, as in the case described herein. Given its rarity, as well as the clinical and radiological difficulties in differentiating these lesions, special attention must be paid to differential diagnoses, especially in cases of discrepancies between the tumor histology and IHC or lack of correlation between image and clinical condition. 